\title{
DNA Optical Detection Based on Porous Silicon Technology: from Biosensors to Biochips
}

\section{Luca De Stefano ${ }^{1, *}$, Paolo Arcari ${ }^{2}$, Annalisa Lamberti ${ }^{2}$, Carmen Sanges ${ }^{2}$, Lucia Rotiroti ${ }^{1,3}$,} Ilaria Rea ${ }^{1,4}$ and Ivo Rendina ${ }^{1}$

${ }^{1}$ Institute for Microelectronics and Microsystems - Unit of Naples - National Council of Research, Via P. Castellino 111, 80131 Napoli, Italy (E-mail: luca.destefano@na.imm.cnr.it)

${ }^{2}$ Department of Biochemistry and Medical Biotechnologies, University of Naples "Federico II", Via S. Pansini 5, 80131 Napoli, Italy.

${ }^{3}$ Department of Organic Chemistry and Biochemistry, University of Naples "Federico II", Via Cinthia, 80128 Napoli, Italy

${ }^{4}$ Department of Physical Sciences, University of Naples "Federico II", Via Cinthia, 80126 Naples, Italy

* Author to whom correspondence should be addressed. E-mail: luca.destefano@na.imm.cnr.it

Received: 15 February 2007 / Accepted: 26 February 2007 / Published: 28 February 2007

\begin{abstract}
A photochemical functionalization process which passivates the porous silicon surface of optical biosensors has been optimized as a function of the thickness and the porosity of the devices. The surface modification has been characterized by contact angle measurements. Fluorescence measurements have been used to investigate the stability of the DNA single strands bound to the nanostructured material. A dose-response curve for an optical label-free biosensor in the 6-80 $\mu \mathrm{M}$ range has been realized.
\end{abstract}

Keywords: Optical Biosensors, Porous Silicon, Biochip.

\section{Introduction}

Biosensors are nowadays technological hot topics due to the possible applications in social interest fields such as medical diagnostic and health care, monitoring of environmental pollutants, home and defence security [1]. Besides the signal generated by the sensing device, the biosensor is constituted by the molecular recognition element and the transducer material. The molecular recognition element can be a biological molecule, such as DNA single strand, proteins, enzymes, or a biological system, such as 
membrane, cell, and tissues: in this way, the sensing mechanism takes advantage of the natural sensitivity and specificity of the biomolecular interactions. Optical transduction is more and more used since photonic devices could be small, lightweight and thus portable due to the integrability of all optical components. Furthermore, optical devices do not require electric contacts. Fluorescence is by far the most used optical signalling method but a wide-use sensor can not be limited by the labelling of the probe nor the analyte, since this step is not always possible [2]. Reagentless optical biosensors are monitoring devices which can detect a target analyte in a heterogeneous solution without the addition of anything than the sample. In the fields of genomics and proteomics this is a straightforward advantage since it allows real-time readouts and, thus, very high throughoutputs analysis. A label free optical biosensor can be realized by integrating the biological probe with a signalling material which directly transduces the molecular recognition event into an optical signal. Recently, lot of theoretical and experimental work, concerning the worth noting properties of nanostructured porous silicon (PSi) in chemical and biological sensing, has been reported, showing that, due to its morphological and physical properties, PSi is a very versatile sensing platform [3, 4]. PSi is an available, low cost material, completely compatible with VLSI and micromachining technologies, so that it could usefully be employed in the fabrication of micro-opto-electric-mechanical system and smart sensors. PSi is also very used as photonic material due to the possibility of fabricating high quality optical structures, either as single layers, like Fabry-Perot interferometers [5], or multilayers, such as Bragg [6] or rugate filters [7]. A key feature for a recognition transducer is a large surface area: PSi has a porous structure with a specific area up to $200-500 \mathrm{~m}^{2} \mathrm{~cm}^{-3}$, so that it can be very sensitive to the presence of biochemical species which penetrate inside the pores. Unfortunately, the surface of the "as etched" PSi is highly hydrophobic so that aqueous solution can not infiltrate the sponge like matrix. A proper passivation process must be applied to stabilise the surface and to covalently link the bioprobe [8]. The development of PSi based biosensor arrays critically depends on the surface functionalization process and how it is compatible with the microfabrication technologies. In a recently published article, we have exploited a photochemical functionalization process of the PSi surface to covalently bind DNA single strands ( $s$ DNA) and we have also demonstrate that the device works as an all optical biosensor for $s s$ DNA- $c$ DNA interactions [9]. In the present work, we have optimised the functionalization process by investigating the role of thickness and porosity of the PSi chip, the time exposure to ultraviolet (UV) light and also the concentration of the ssDNA solution. Fluorescent measurements have been used to test the chip stability against the washing in aqueous solutions.

\section{Experimental Section}

In this study we used as optical transducer a porous silicon layer of fixed thickness and porosity: from an optical point of view, this structure acts as a Fabry-Perot interferometer. To study the influence of porous silicon physical parameters, we have fabricated several layers, obtained by electrochemical etch in a $\mathrm{HF} / \mathrm{EtOH}(3: 7)$ solution, at room temperature and dark light. Highly doped $\mathrm{p}^{+}$-silicon, <100> oriented, $0.005 \Omega \mathrm{cm}$ resistivity, $400 \mu \mathrm{m}$ thick was used. The PSi samples were characterised by variable angle spectroscopic ellipsometry [10]. Etching times and anodic current densities have been reported in Table 1. 
Table 1. PSi layers physical characteristics and fabrication parameters.

\begin{tabular}{|c|c|c|c|c|}
\hline \multirow{2}{*}{$\begin{array}{c}\text { Current Density } \\
\left(\mathrm{mA} / \mathrm{cm}^{2}\right)\end{array}$} & $\begin{array}{c}\text { Porosity } \\
(\%)\end{array}$ & $\begin{array}{c}\text { Etch Rate } \\
(\mathrm{nm} / \mathrm{s})\end{array}$ & $\begin{array}{c}\text { Thickness } \\
(\mu \mathrm{m})\end{array}$ & $\begin{array}{c}\text { Etch Time } \\
(\mathrm{s})\end{array}$ \\
\hline \multirow{2}{*}{50} & \multirow{2}{*}{60} & \multirow{2}{*}{193} & 2.0 & 10.4 \\
\cline { 4 - 5 } & & & 4.0 & 20.7 \\
\cline { 4 - 5 } & \multirow{2}{*}{70} & \multirow{2}{*}{247} & 6.0 & 31.1 \\
\cline { 4 - 5 } & & & 2.0 & 8.1 \\
\cline { 4 - 5 } & \multirow{2}{*}{125} & \multirow{2}{*}{137} & 6.0 & 24.3 \\
\cline { 4 - 5 } & \multirow{2}{*}{150} & \multirow{2}{*}{137} & 2.0 & 14.0 \\
\cline { 4 - 5 } & & & 6.0 & 29.2 \\
\hline
\end{tabular}

The photo-activated chemical modification of PSi surface was based on the UV exposure of a solution of alkenes which bring some carboxylic acid groups. The PSi chip has been pre-cleaned in an ultrasonic acetone bath then washed in deionized water. After dried in $\mathrm{N}_{2}$ stream, it has been immediately covered with $10 \% \mathrm{~N}$-hydroxysuccinimide ester (UANHS) solution in $\mathrm{CH}_{2} \mathrm{Cl}_{2}$. The UANHS was house synthesised as described in literature [11]. This treatment results in covalent attachment of UANHS to the porous silicon surface. The chip was then washed in dichloromethane in an ultrasonic bath for $10 \mathrm{~min}$ to remove any adsorbed alkene from the surface. The carboxyl-terminated monolayer covering the PSi surface works as a reactive substrate for the chemistry of the subsequent attachment of the DNA sequences. DNA single strands in a HEPES solution $10 \mathrm{mM}(\mathrm{pH}=7.5)$ have been incubated overnight.

FT-IR spectroscopy (Thermo - Nicholet NEXUS) has been used to verify the efficiency of the reaction. After the chemical functionalization we have also quantitatively measured the efficiency of the binding between the DNA and the porous silicon surface using a fluorescent DNA probe (5'GGACTTGCCCGAATCTACGTGTCCA3', Primm) labelled with a proper chromophore group (Fluorescein CY3.5, the absorption peak is at $581 \mathrm{~nm}$ and the emission is at $596 \mathrm{~nm}$ ). Fluorescence images were recorded by a Leica Z16 APO fluorescence macroscopy system. The fluorescent chips have been dialyzed overnight, first in water and then in a HEPES solution, at room temperature to assess the binding between the bioprobe and the PSi surface.

Contact angle measurements have been performed by using a KSV Instruments LTD CAM 200 Optical Contact Angle Meter.

The reflectivity measurements have been performed by a very simple experimental set-up: a tungsten lamp (400 $\mathrm{nm}<\lambda<1800 \mathrm{~nm}$ ) illuminates, through an optical fiber and a collimator, the sensor and the reflected beam is collected by an objective, coupled into a multimode fiber, and then directed in an optical spectrum analyser (Ando, AQ6315A). The reflectivity spectra have been measured with a resolution of $0.5 \mathrm{~nm}$. 


\section{Results and Discussion}

Infrared spectroscopy is a powerful tool in surfaces characterisation: it is fast, accurate and could also performe quantitative determinations. We have investigated all the PSi monolayers before and after the photochemical passivation process since we were worried about the functionalization efficiency of thicker samples. A thicker layer can adsorb more bioprobes than a thinner one but the UV exposure could also be less effective, due to light absorption by PSi at those frequencies. Our measurements demonstrate that up to $6 \mu \mathrm{m}$ thick PSi monolayer, a complete passivation and functionalization of the surface can be obtained by exposing for a sufficiently long time the sample: in Figure 1A are shown the FT-IR spectra of the PSi monolayer as etched and after three different times of exposure to UV light.
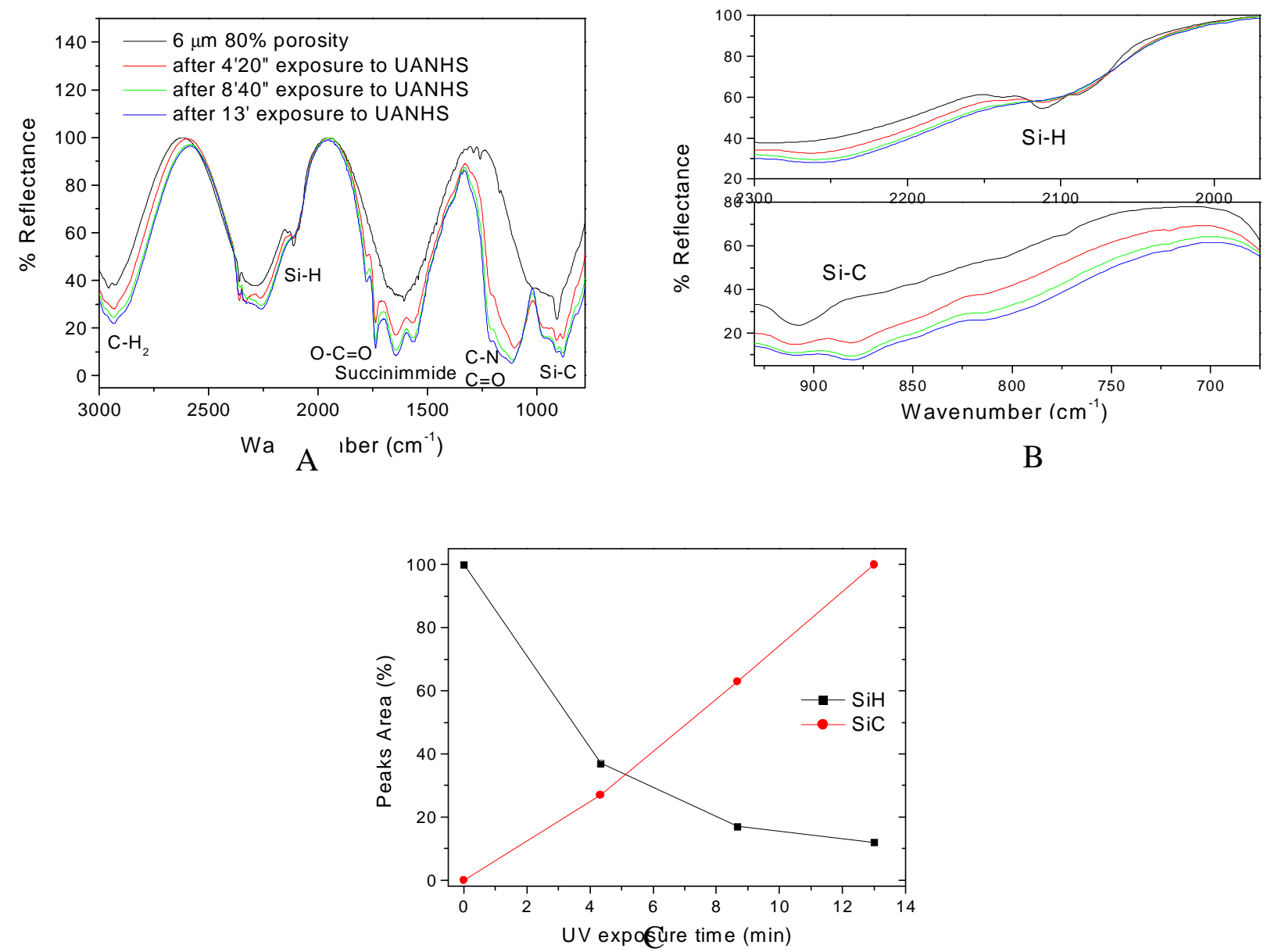

Figure 1. A) FT-IR spectra of the PSi monolayer as etched and after three different times of exposure to UV light. B) Particulars of the Si-H bond and Si-C bond peaks at 2100 and $880 \mathrm{~cm}^{-1}$, respectively. C)

Peaks area as function of the exposure time: the reaction yield increases monotonically with the exposure time. 
The characteristic peaks of the Si-H bonds (at $2100 \mathrm{~cm}^{-1}$ ) progressively disappear while the amide I band (at $1634 \mathrm{~cm}^{-1}$ ) and the Si-C peak (at $880 \mathrm{~cm}^{-1}$ ) become more and more evident. Figure $1 \mathrm{~B}$ is an enlargement of the characteristic peaks of these resonances. The reaction yield increases quite monotonically with the exposure time, as it can be seen in Figure 1C. Thinner samples can be functionalised in faster exposure times, but the overall volume available to adsorb bioprobes is drastically reduced.

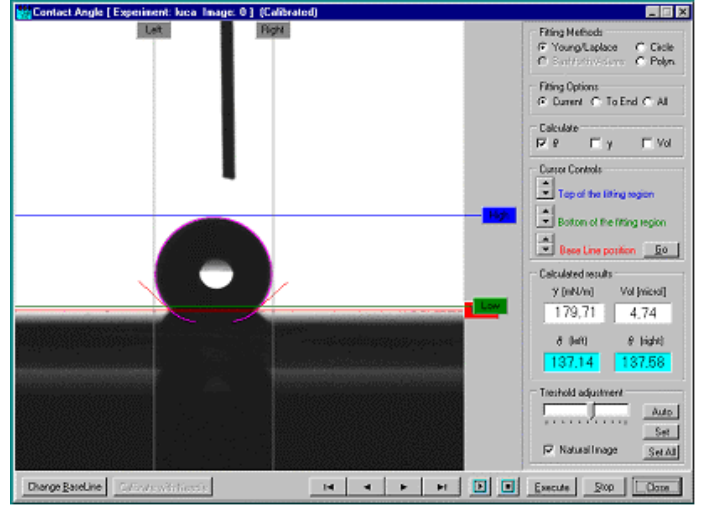

A

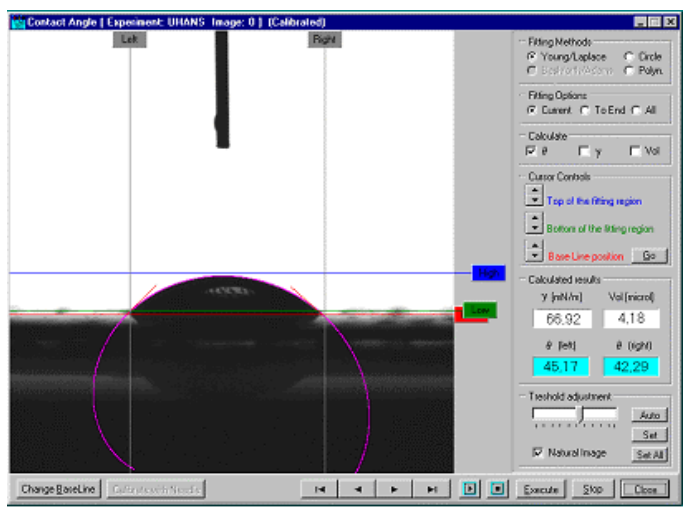

B

Figure 2. Characterisation of the PSi surface by contact angles measurements. A) The PSi as etched shows a clearly hydrophobic behaviour. B) After the passivation process the PSi surface is hydrophilic thus allowing the penetration of biological solution.

The surface passivation is also well evident by contact angle measurements: the hydrogenated PSi surface is highly hydrophobic, due to the presence of the Si-H bonds, so that a drop of deionised water exploits a high surface tension which prevents its diffusion in the pores. This behaviour is well depicted in Figure 2A: the contact angle is $137 \pm 1$ degree, averaging the left and right values of three different drops having the same volumes, and the estimated surface tension is $179 \mathrm{mN} / \mathrm{m}$. After the photoreaction with the UANHS, the $\mathrm{Si}-\mathrm{H}$ bonds are replaced by the $\mathrm{Si}-\mathrm{C}$ bonds and the PSi surface shows a hydrophilic behaviour, as shown in Figure 2B: the drop spreads on the surface and the contact angle drastically decreases to $43 \pm 3$ degree and also the surface tension is lowered to $67 \mathrm{mN} / \mathrm{m}$. In this condition, an aqueous solution can be infiltrated in the nanostructured layer.

To test the stability of the covalent bonding between the organic linker layers, which homogeneously cover the PSi surface, and the biological probes we have used a fluorescent DNA single strand as an optical tracer. After the chemical bonding of the labelled ssDNA, the chip was observed by the fluorescence macroscopy system. Under the light of the $100 \mathrm{~W}$ high-pressure mercury source, we have found a high and homogeneous fluorescence on the whole chip surface which still remains bright even after two overnight dialysis washings in a HEPES solution and in deionised water, as it can be seen in Figures 3 A, B, and C. We have also studied the yield of the chemical functionalization by spotting different concentrations of the fluorescent $s s \mathrm{DNA}$ and measuring the fluorescence intensities of the images before and after the washings. The results reported in Figure 4 confirm the qualitative findings of Figure 3: the fluorescent intensities decrease but remain of the same order of magnitude. From this 
graph we can also estimate the concentration of the DNA probe which saturates the binding sites available.

The PSi optical biosensors measure the change in the average refractive index of the device: when a bioconjugation event takes place, the refractive index of the molecular complex changes and the interference pattern on output is thus modified. The label free optical monitoring of the $s s$ DNA-cDNA hybridization is simply the comparison between the optical spectra of the porous silicon layer after the UANHS and probe immobilization on the chip surface and after its hybridization with the $c$ DNA. Each step of the chip preparation increases the optical path in the reflectivity spectrum recorded, due to the substitution of the air into the pores by the organic and biological compounds. The interaction of the ssDNA with its complementary sequence has been detected as a fringes shift in the wavelengths, which corresponds to a change in the optical path. Since the thickness $d$ is fixed by the physical dimension of the PSi matrix, the variation is clearly due to changes in the average refractive index.

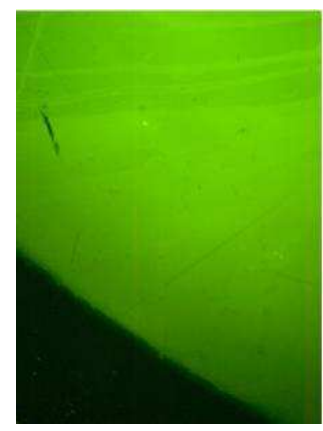

A

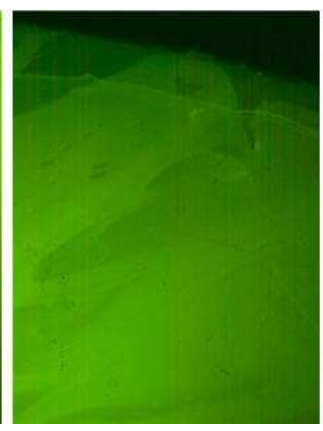

B

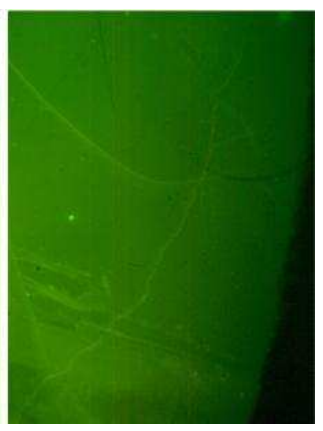

C

Figure 3: A) Fluorescence of the chip surface after the binding of the labelled $s s \mathrm{DNA}$; B) after the overnight dialysis in HEPES solution; C) after the overnight dialysis in deionised water.

In Figure 5A the reflectivity spectra of the PSi layer for different $c$ DNA concentration are reported, while in Figure 5B a dose-response curve is reported. A control measurement has been made using a $n c$ DNA sequence: a very small shift (less than $2 \mathrm{~nm}$ ) has been recorded in the reflectivity spectrum respect to the one obtained after the probe linking.

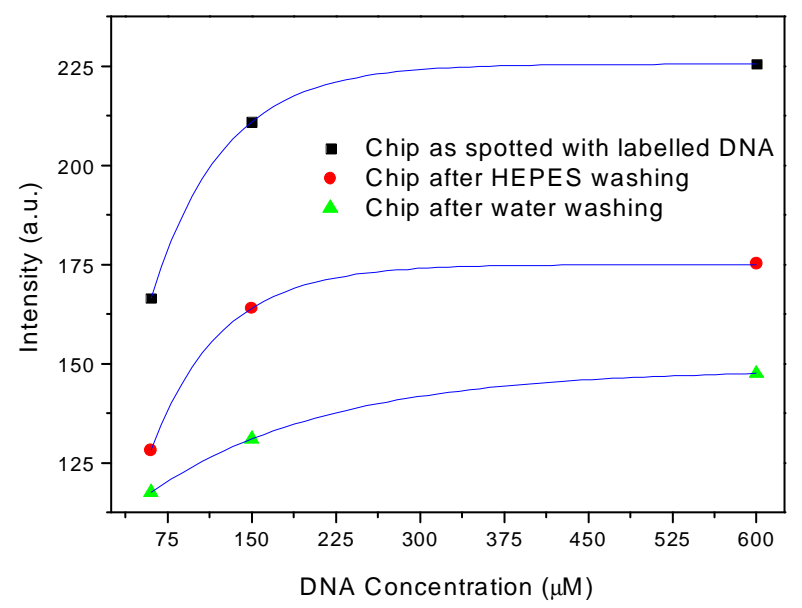

Figure 4. Fluorescence intensities of the chip surface after the binding of the labelled ssDNA and the two overnight dialysis as a function of the $c$ DNA concentration. 
The sensor response has been fitted by a monoexponential growth model, $y=A-B e^{-k x}$, where $A$ is the offset, $B$ the amplitude, $k$ the rate and $y^{\prime}=B k$ the limiting sensitivity, i.e. the sensitivity in the limit of zero ligand concentration. In this case, we obtained for this parameter the value of $1.1(0.1) \mathrm{nm} / \mu \mathrm{M}$, which corresponds to a limit of detection of $90 \mathrm{nM}$ for a system able to detect a wavelength shift of 0.1 nm.

A

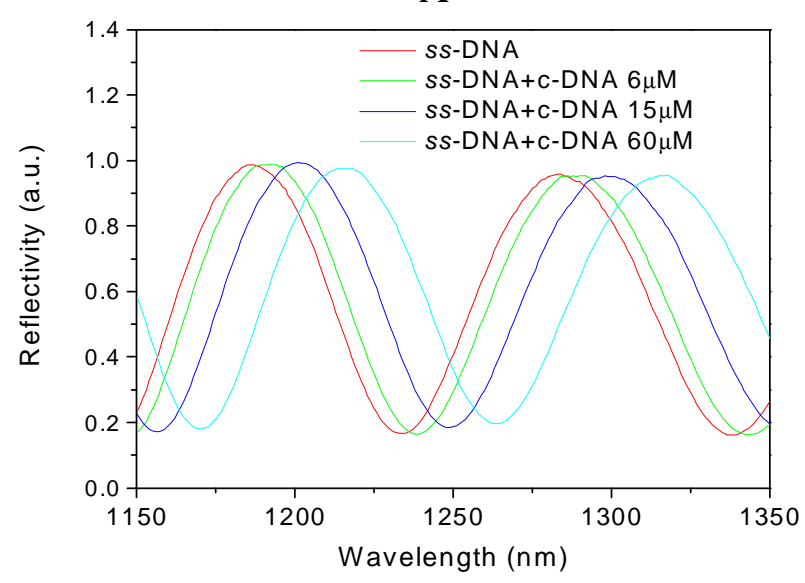

B

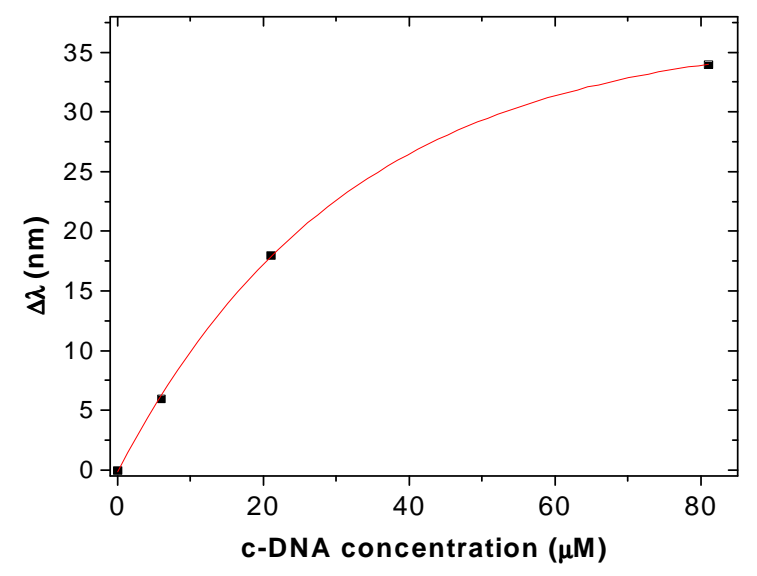

Figure 5. A) Fringes shifts due to $s s$ DNA-cDNA interaction. B) Dose-response curve as a function of the cDNA concentration.

In conclusions, we have optimized the PSi surface functionalization process by investigating the role of chip thickness and porosity, on exposure to ultraviolet (UV) light for different times interval and also for different concentration of the ssDNA probe. Fluorescent measurements have confirmed the chip stability against the washing in aqueous solutions. These results will be very useful in the design and realisation of a PSi based label free optical biochip.

\section{Acknowledgements}

Authors wish to acknowledge dr. Andrea M. Rossi of National Institute of Metrological Research, Turin, Italy, for helpful discussions and Prof. N. Scaramuzza and dr. M. Giocondo of University of Calabria, Cosenza, Italy, for contact angle measurements. This work is partially supported by the FIRB Italian project RBLA033WJX_005.

\section{References and Notes}

1. Optical Biosensors, Eds. F.S. Ligler and C.A. Rowe Taitt, Elsevier, Amsterdam, The Netherlands, 2004.

2. Salitermkan, S. S. Fundamentals of BioMEMS and Medical Microdevices; Wiley-Interscience, SPIE PRESS (Bellingham, Washington USA), 2006.

3. Lin, V. S. Y.; Motesharei, K.; Dancil, K. P. S.; Sailor, M. J.; Ghadiri, M. R., A porous siliconbased optical interferometric biosensor, Science 1997, 278, 840-843. 
4. De Stefano, L.; Moretti, L.; Rendina, I.; Rossi, A. M., Time-resolved sensing of chemical species in porous silicon optical microcavity, Sensor and Actuators B 2004, 100, 168-172.

5. Dancil, K. -P. S.; Greiner, D. P.; Sailor, M. J., A Porous Silicon Optical Biosensor: Detection of Reversible Binding of IgG to a Protein A-Modified Surface, J. Am. Chem. Soc. 1999, 121, $7925-$ 7930.

6. Snow, P. A.; Squire, E. K.; Russel, P. St. J.; Canham, L. T., Vapor sensing using the optical properties of porous silicon Bragg mirrors, J. Appl. Phys. 1999, 86, 1781-1784.

7. Lorenzo, E.; Oton, C. J.; Capuj, N. E.; Ghulinyan, M.; Navarro-Urrios, D.; Gaburro, Z.; Pavesi, L., Porous silicon-based rugate filters, Appl. Opt. 2005, 44, 5415-5421.

8. Ouyang, H.; Chrtistophersen, M.; Viard, R.; Miller, B. L.; Fauchet, P. M., Macroporous silicon microcavities for macromolecule detection, Adv. Funct. Mater. 2005, 15, 1851-1859.

9. De Stefano, L.; Rotiroti, L.; Rea, I.; Rendina, I.; Moretti, L.; Di Francia, G.; Massera, E.; Lamberti, A.; Arcari, P.; Sangez, C., Porous silicon-based optical biochips, Journal of Optics A: Pure and Applied Optics 2006, 8, S540-S544.

10. Wongmanerod, C.; Zangooie, S.; Arwin, H., Determination of pore size distribution and surface area of thin porous silicon layers by spectroscopic ellipsometry, Applied Surface Science 2001, 172, 117-125.

11. Yin, H.B.; Brown, T.; Gref, R.; Wilkinson, J.S.; Melvin, T., Chemical modification and micropatterning of Si(100) with oligonucleotides, Microelectronic Engineering 2004, 73-74, 830836.

(C) 2007 by MDPI (http://www.mdpi.org). Reproduction is permitted for noncommercial purposes. 